\title{
Tougu Xiaotong capsule promotes chondrocyte autophagy by regulating the Atg12/LC3 conjugation systems
}

\author{
XIHAI LI ${ }^{1}$, FAYUAN LIU ${ }^{1}$, WENNA LIANG ${ }^{2}$, HONGZHI YE ${ }^{1}$, HUITING LI $^{3}$, FANGRONG YU ${ }^{3}$, JIASHOU CHEN ${ }^{3}$, \\ WENLIE CHEN $^{1}$, RUHUI LIN ${ }^{3}$, CHUNSONG ZHENG ${ }^{1}$, GUANGWEN WU $^{1}$, HUIFENG XU ${ }^{1}$ and XIANXIANG LIU ${ }^{1}$ \\ ${ }^{1}$ Academy of Integrative Medicine, ${ }^{2}$ Research Base of Traditional Chinese Medicine Syndrome, ${ }^{3}$ Fujian Key Laboratory of \\ Integrative Medicine on Geriatrics, Fujian University of Traditional Chinese Medicine, Fuzhou, Fujian 350122, P.R. China
}

Received January 3, 2014; Accepted May 26, 2014

DOI: $10.3892 /$ ijmm.2014.1794

\begin{abstract}
We have previously reported that Tougu Xiaotong capsule (TXC) inhibits tidemark replication and cartilage degradation by regulating chondrocyte autophagy in vivo. Autophagy, a cell protective mechanism for maintaining cellular homeostasis, has been shown to be a constitutively active and protective process for chondrocyte survival. However, it remains unclear whether TXC promotes chondrocyte autophagy by regulating the autophagy-related (Atg)12/microtubuleassociated protein 1 light chain 3 (LC3) conjugation systems. Thus, in the present study, we investigated the effects of TXC on primary chondrocytes treated with cobalt chloride $\left(\mathrm{CoCl}_{2}\right)$. We found that $\mathrm{CoCl}_{2}$ induced a decrease in chondrocyte viability and the autophagosome formation of chondrocytes, indicating that $\mathrm{CoCl}_{2}$ induced autophagic death in a dose- and time-dependent manner. To determine the effects of TXC on $\mathrm{CoCl}_{2}$-exposed chondrocytes, we assessed cell viability by MTT assay. Our results revealed that TXC enhanced the viability of $\mathrm{CoCl}_{2}$-exposed chondrocytes. To gain insight into the mechanisms responsible for the enhancing effects of TXC on $\mathrm{CoCl}_{2}$-exposed chondrocytes, the expression of Atg genes was assessed in chondrocytes exposed to $\mathrm{CoCl}_{2}$ and treated with or without TXC. The results revealed that the expression of beclin 1, Atg3, Atg5, Atg7, Atg10, Atg12 and LC3 II/LC3 I in the chondrocytes treated with TXC increased, compared to that in the untreated chondrocytes. In addition, ultrastructural analysis indicated that treated chondrocytes contained more autophagosomes than the untreated cells, suggesting that TXC increased the formation of autophagosomes in the chondrocytes to clear the $\mathrm{CoCl}_{2}$-induced autophagic death. Therefore, these data suggest that TXC is a potential therapeutic agent for the reduction of cartilage degradation that occurs in osteoarthritis.
\end{abstract}

Correspondence to: Dr Xianxiang Liu, Academy of Integrative Medicine, Fujian University of Traditional Chinese Medicine, 1 Huatuo, University Town, Minhou Shangjie, Fuzhou, Fujian 350122, P.R. China

E-mail: liuxianxiang@163.com

Key words: autophagy, chondrocyte, Tougu Xiaotong capsule, osteoarthritis

\section{Introduction}

Osteoarthritis (OA), a highly prevalent joint disease, exhibits a number of histological characteristics, including a gradual degradation of the extracellular matrix (ECM) and reduced cartilage cellularity, as well as a disruption of the articular cartilage surface, belonging to the GuBi of Traditional Chinese Medicine (TCM) (1-3). Chondrocytes, the only cell population of the articular cartilage, are capable of responding to structural changes in the surrounding ECM by maintaining the dynamic equilibrium between production of the ECM and its enzymatic degradation; however, the capacity of chondrocytes to regenerate the normal ECM architecture is limited and declines with aging due to abnormal responsiveness to anabolic stimuli and cell death (4,5). A number of factors may be involved in the development of OA; however, one of the most important risk factors is cell death. Cell death diminishes the ability of cells to proliferate, mainly due to an increase in apoptosis, which is thought to be a major cause of chondrocyte depletion during OA progression. Several studies have demonstrated that another type of cell death, autophagy, is involved in chondrocyte depletion during OA progression (6-8).

Autophagy, a cellular homeostatic mechanism, plays an important role in nutrient and energy regulation, and in targeting dysfunctional and altered cytosolic macromolecules, membranes and organelles for delivery to lysosomes for recycling and degradation (9-11). At the cellular level, failure of autophagy leads to the increased production of abnormal gene expression, reactive oxygen species, and may cause cell death (12). The consequences of autophagy failure at the tissue and organismal level are abnormal skeletal development, cardiomyopathies, neurodegeneration and premature death $(7,13,14)$. The mammalian target of rapamycin (mTOR), an important suppressor of autophagy, functions upstream of the autophagy-related (Atg) proteins and is crucially regulated by multiple upstream signaling pathways involving adenosine monophosphate (AMP)-activated protein kinase and phosphoinositide 3 (PI3)-kinase/Akt $(15,16)$. In articular cartilage, which is characterized by a very low rate of cell turnover, autophagy appears to be essential to maintain cellular integrity, survival and function $(7,8)$. Previous studies have verified that autophagy is a constitutively active and apparently protective process for the homeostatic state in normal cartilage (17). A 
reduced expression of Atg genes has been observed in OA in humans and mice and is accompanied by an increase in chondrocyte apoptosis, indicating a protective and survivalpromoting function of autophagy $(7,8,17)$.

Tougu Xiaotong capsule (TXC; Medical License number: MINZHIZI Z20100006) is composed of a combination of 4 natural products, including Morindae officinalis, Radix Paeoniae Alba, Rhizoma Chuanxiong and Sarcandra glabra. According to the theories of TCM, these natural products mixed together confer the TXC properties of nourishing Shen, filling in Sui, supplementing Jing, dredging the meridians and collaterals to limber the joints and strengthen bones and tendons. TXC has been widely used for the therapy of OA in the Second People's Hospital affiliated to Fujian University of TCM for 2 decades and has been shown to control pain and improve dysfunction in patients with OA (18). We have previously reported that TXC inhibits tidemark replication and cartilage degradation by the regulation of chondrocyte autophagy (19). However, the precious molecular and cellular mechanisms responsible for the effects of TXC on the regulation of chondrocyte autophagy remain largely unknown. Thus, the aim of this study was to establish a proof-of-principle that the pharmacological enhancement of autophagy may be an effective therapeutic approach for OA by regulating the Atg12/microtubule-associated protein 1 light chain 3 (LC3) conjugation systems. Our data demonstrate that TXC reduces the severity of chondrocyte damage, at least in part by activating autophagy, suggesting that TXC promotes chondrocyte autophagy, contributing to the regulation of cartilage homeostasis.

\section{Materials and methods}

TXC extracts and fingerprint analysis. TXC herbs were dried for $24 \mathrm{~h}$ in an air-circulating oven at $50^{\circ} \mathrm{C}$ and then shredded and crushed to an appropriate particle size in a high-speed rotary cutting mill (ZN-400A; Zhongnan Pharmaceutical Machinery Factory, Changsha, China). According to the proportion of TXC (Morinda officinalis:Radix Paeoniae Alba:Rhizoma Chuanxiong:Sarcandra glabra = 2:2:1:1), $108 \mathrm{~g}$ of herbal powder were extracted with 1.51 distilled water by reflux for 2 times, $2 \mathrm{~h}$ per times. The filtrate withdrawn from the TXC was evaporated using a rotary evaporator (RE-2000; Shanghai Yarong Biochemical Instrument Factory, Shanghai, China) and was then dried to constant weight in a vacuum drying oven (DZF-300; Shanghai Hengke Electronic Technology Co., Ltd., Shanghai, China). The TXC extracts were dissolved in Dulbecco's modified Eagle's medium (DMEM; Gibco, Grand Island, NY, USA) as a $20 \mathrm{mg} / \mathrm{ml}$ stock solution.

The quality control of the TXC extracts was analyzed by high performance liquid chromatography (HPLC)fingerprint on an Agilent 1200 HPLC system (Agilent, Santa Clara, CA, USA) using an Ultimate $^{\mathrm{TM}}$ XB-C18 column $(4.60 \times 250.00 \mathrm{~mm}$, $5 \mu \mathrm{m}$, Welch Materials, Inc., Ellicott City MD, USA). The conditions for analysis were methanol- $0.1 \%$ phosphoric acid as a mobile phase and a detection wavelength at $277 \mathrm{~nm}$, a flow rate of $1 \mathrm{ml} / \mathrm{min}$ and a column temperature of $30^{\circ} \mathrm{C}$. The gradient procedure was as follows: $5 \% \mathrm{~A}$ at $0-5 \mathrm{~min}, 5-20 \% \mathrm{~A}$ at 5-10 $\mathrm{min}, 20-42 \% \mathrm{~A}$ at $15-25 \mathrm{~min}, 42-65 \% \mathrm{~A}$ at $25-40 \mathrm{~min}$, $65-80 \% \mathrm{~A}$ at $40-55 \mathrm{~min}$ and $80-100 \% \mathrm{~A}$ at $55-70 \mathrm{~min}$. Paeoniflorin, ferulic acid, isofraxidin and rosmarinic acid
(National Institute for Pharmaceutical and Biological Products Control, Beijing, China) were used as standard substances.

Chondrocyte isolation and culture. Animal use protocols were performed according to the Guide for the Care and Use of Laboratory Animals approved by the Animal Care and Use Committee of Fujian University of TCM. Chondrocytes were collected from the knee articular cartilage of 4-weekold Sprague-Dawley (SD) rats (Shanghai Slack Laboratory Animal Co., Shanghai, China). Chondrocytes were isolated using $0.2 \%$ type II collagenase (Sigma, St. Louis, MO, USA) in pH 7.4 magnesium- and calcium-free phosphate-buffered saline (PBS; Sigma) for $1 \mathrm{~h}$ at $37^{\circ} \mathrm{C}(20)$. Chondrocytes were resuspended in low-glucose DMEM (Gibco) supplemented with $10 \%$ fetal calf serum (Gibco), streptomycin $(100 \mu \mathrm{g} / \mathrm{ml})$ and penicillin $(100 \mathrm{U} / \mathrm{ml})$, and seeded in monolayer at a density of $5 \times 10^{5}$ cells $/ \mathrm{cm}^{2}$. The chondrocytes used in this study were subjected to the second passage cells, identified by type II collagen immunohistochemistry and scanning electron microscopy (SEM). Cobalt chloride $\left(\mathrm{CoCl}_{2}\right)$ powder (Sigma) was dissolved in DMSO as a $1 \mathrm{mM}$ stock solution. The second-passage chondrocytes cultured till approximately $80 \%$ confluency were treated with $100 \mu \mathrm{M}$ of $\mathrm{CoCl}_{2}$ with or without TXC for $48 \mathrm{~h}$.

Assessment of cell viability by MTT assay. The cells were seeded in a 96-well plate at a density of $1.2 \times 10^{4} \mathrm{cells} / \mathrm{cm}^{2}$ and allowed to adhere overnight, then treated with either $\mathrm{CoCl}_{2}$ $(0,50,100,200,300,400 \mu \mathrm{M})$ for $24 \mathrm{~h}$ or with $100 \mu \mathrm{M}$ of $\mathrm{CoCl}_{2}$ for $6,12,24,48,72 \mathrm{~h}$ and either TXC $(0,50,100,200$, $300,400 \mu \mathrm{g} / \mathrm{ml})+100 \mu \mathrm{M} \mathrm{CoCl}_{2}$ for $48 \mathrm{~h}$ or with $200 \mu \mathrm{g} / \mathrm{ml}$ of TXC $+100 \mu \mathrm{M} \mathrm{CoCl}_{2}$ for 6, 12, 24, 48 and $72 \mathrm{~h}$. Following treatment, $20 \mu 1$ MTT stock solution $(5 \mathrm{mg} / \mathrm{ml})$ were added to each well, and the cells were incubated at $37^{\circ} \mathrm{C}$ for $4 \mathrm{~h}$. Thereafter, the medium was aspirated followed by the addition of $200 \mu 1 \mathrm{DMSO}$, and the cells were shaken for $10 \mathrm{~min}$. The color formed was determined by an ELISA plate reader (EXL 800; BioTek, Winooski, VT, USA).

Reverse transcription-polymerase chain reaction (RT-PCR). Total RNA was extracted from the chondrocytes using TRIzol reagent (Invitrogen, Carlsbad, CA, USA). Reverse transcription was performed using random primers and Superscript $^{\mathrm{TM}}$ III (Invitrogen). PCR reactions were conducted on an ABI Prism 7900HT Fast Real-Time PCR System (Applied Biosystems, Foster City, CA, USA). Primers for the amplifications were designed as follows: hypoxia-inducible factor- $1 \alpha$ (HIF-1 $\alpha)$ forward, 5'-GCA TCT CCA CCT TCT ACC C-3' and reverse, 5'-TTC TGC TCC ATT CCA TCC T-3', 386 bp; beclin 1 forward, 5'-GCT CAG TAC CAG CGA GAA TA-3' and reverse, 5'-GTC AGG GAC TCC AGA TAC GA-3', $350 \mathrm{bp}$; mTOR forward, 5'-GGA CGG TGT AGA ACT TGG A-3' and reverse, 5'-GAG ATG TCG CTT GCT TGA T-3', $230 \mathrm{bp}$; Atg3 forward, 5'-GGA GGC TAT CAT TGA AGA AG-3' and reverse, 5'-TGG GAG GTG AGG ATG GTT T-3', 481 bp; Atg5 forward, 5'-ACG CTG GTA ACT GAC AAA G-3' and reverse, 5'-CAC ATG ACA TAA AGT GAG CC-3', 250 bp; Atg7 forward, 5'-TGG GAG AAG AAC CAG AAA GG-3' and reverse, 5'-TCA CGG GAT TGG AGT AGG AG-3', 280 bp; Atg10 forward, 5'-GTG CCC GTT CTG TAC TTT AGG-3' and 
reverse, 5'-TCA TTT GTC TTG CAG GGA TGT-3', 188 bp; Atg12 forward, 5'-GAG ACA CTC CCA TAA TGA A-3' and reverse, 5'-GTA GGA CCA GTT TAC CAT C-3', 207 bp; LC3 I forward, 5'-CTT CGC CGA CCG CTG TAA-3' and reverse, 5'-ATC CGT CTT CAT CCT TCT CCT G-3', 287 bp; LC3 II forward, 5'-CTA ACC AAG CCT TCT TCC TCC-3' and reverse, 5'-GGT GCC TAC GTT CTG ATC TGT G-3', 261 bp; $\beta$-actin forward, 5'-CAC CCG CGA GTA CAA CCT TC-3' and reverse, 5'-CCC ATA CCC ACC ATC ACA CC-3', 207 bp.

Western blot analysis. Total protein was extracted from the chondrocytes using RIPA buffer, and protein concentrations were determined using a Bio-Rad protein assay (Bio-Rad, Hercules, CA, USA). Total protein $(50 \mu \mathrm{g})$ was fractionated by SDS-PAGE, and transferred onto a PVDF membrane (Invitrogen). The PVDF membrane was blocked with 5\% non-fat milk and incubated with antibodies to HIF-1 $\alpha$, beclin 1 , mTOR, Atg3, Atg7, Atg12, LC3 I/II and $\beta$-actin (Santa Cruz Biotechnology, Santa Cruz, CA, USA). The blots were developed using a horseradish peroxidase-conjugated secondary antibody (Beyotime Institute of Biotechnology, Shanghai, China). Immunoreactive proteins were visualized by western blotting chemiluminescence luminol reagent (Santa Cruz Biotechnology). Immunoblotting band gray values were calculated using the Tocan 190 protein assay system (Bio-Rad).

Transmission electron microscopy (TEM). The chondrocytes were fixed in $3 \%$ glutaraldheyde and $1.5 \%$ paraformaldehyde solution ( $\mathrm{pH} 7.3$ ) at $4^{\circ} \mathrm{C}$ for $24 \mathrm{~h}$, post-fixed with $1 \%$ osmic acid and $1.5 \%$ potassium hexacyanoferrate (II) solution (pH 7.3) at $4^{\circ} \mathrm{C}$ for $2 \mathrm{~h}$, rinsed with water, dehydrated in a graded series of ethanol followed by propylene oxide, kept overnight and embedded in Epon-Araldite resin. Ultrathin sections were obtained using a Leica ultramicrotome and stained with $2 \%$ aqueous uranyl acetate, counterstained with $0.3 \%$ lead citrate and observed under a transmission electron microscope (H7650; Hitachi High-Technologies Corp., Tokyo, Japan).

Statistical analysis. All data were collected from at least 3 independent experiments. Statistical analysis was performed using SPSS 13.0 software. All data are presented as the means \pm standard deviation (SD) and analyzed by the Student's t-test and ANOVA. Statistical significance was set at $\mathrm{P}<0.05$.

\section{Results}

Quality control of TXC. Compared to the spectrogram and chromatographic peak of retention time with the reference substance (Fig. 1A), the composition of TXC was identified (Fig. 1B), and contained paeoniflorin, ferulic acid, isofraxidin and rosmarinic acid.

Identification of chondrocytes. Type II collagen has been identified as the major molecular form of collagen in articular cartilage and is responsible for tensile strength, whereas proteoglycans provide the compressive stiffness necessary for normal articulation and function (5). The second generation of chondrocytes was cultured for 3 days followed by type II collagen immunohistochemical staining. The cytoplasm was stained with brown, representing positive expression in chondrocytes (Fig. 2A
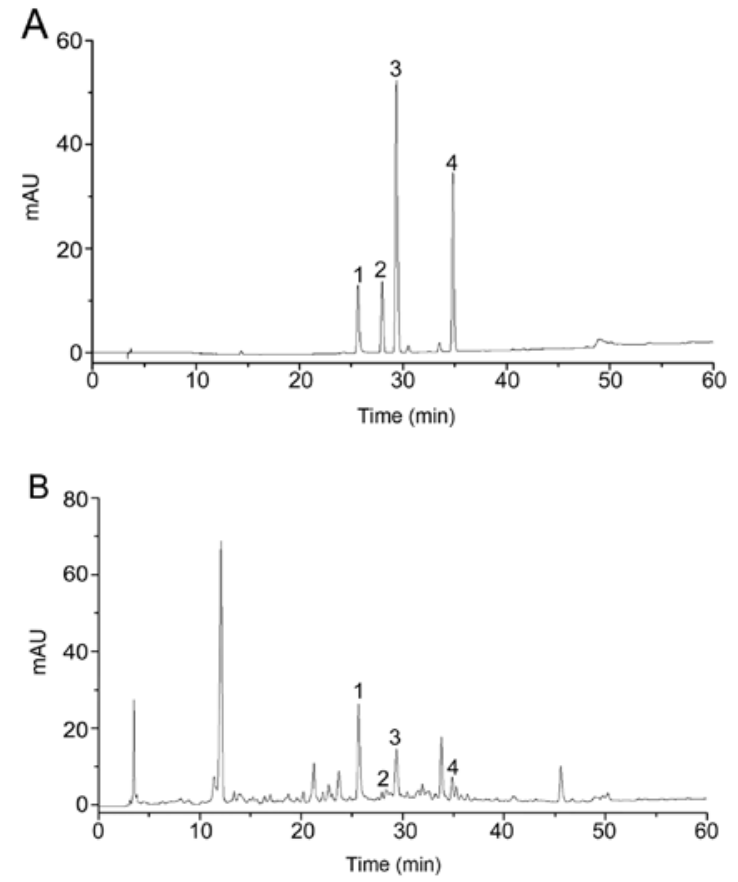

Figure 1. Liquid chromatogram of the reference substance and Tougu Xiaotong capsule (TXC). (A) The liquid chromatogram of the reference substance was composed of 4 peaks, i.e., paeoniflorin (peak 1), isofraxidin (peak 2), ferulic acid (peak 3) and rosmarinic acid (peak 4) . (B) The 4 peaks were also found in the liquid chromatogram of TXC.

and B). The second generation of chondrocytes showed a typical morphology of chondrocytes with a polygonal or spherical shape (Fig. 2C and D). Therefore, we used the second generation of chondrocytes in the subsequent experiments.

TXC increases the cell viability of $\mathrm{CoCl}_{2}$-exposed chondrocytes. $\mathrm{CoCl}_{2}$ is commonly used to activate autophagic death by inducing HIF-1 $\alpha$. To establish the cell model of autophagic death, the chondrocytes were treated with various concentrations and for different periods of time with $\mathrm{CoCl}_{2}$ to determine the effective concentration and treatment duration time by MTT assay. As shown in Fig. 3A, in the cells that were treated with $\mathrm{CoCl}_{2}$ concentrations of $50 \mu \mathrm{M}(68.43 \pm 3.78 \%)$, $100 \mu \mathrm{M}(50.38 \pm 4.12 \%), 200 \mu \mathrm{M}(42.84 \pm 1.95 \%), 300 \mu \mathrm{M}$ $(41.13 \pm 3.20 \%)$ and $400 \mu \mathrm{M}(36.10 \pm 4.89 \%)$ for $24 \mathrm{~h}$, a dosedependent decrease in cell viability was observed compared to the untreated cells $(100 \pm 0.00 \%)(\mathrm{P}<0.01)$. Cell viability gradually decreased with the increase in the duration of treatment in the chondrocytes treated with $100 \mu \mathrm{M} \mathrm{CoCl}_{2}$ for different periods of time (Fig. 3B), suggesting that $\mathrm{CoCl}_{2}$ inhibited cell viability in a dose- and time-dependent manner due to the $\mathrm{CoCl}_{2}$-induced autophagic death.

To explore the effects of TXC on $\mathrm{CoCl}_{2}$-treated chondrocytes, we examined the cell viability of $\mathrm{CoCl}_{2}$-exposed chondrocytes treated with various concentrations of TXC and for different periods of time by MTT assay. As shown in Fig. 3C, the cell viability of the $\mathrm{CoCl}_{2}$-exposed chondrocytes treated with TXC concentrations of $50 \mu \mathrm{g} / \mathrm{ml}(105.65 \pm 3.38 \%)$, $100 \mu \mathrm{g} / \mathrm{ml}(111.38 \pm 3.04 \%), 200 \mu \mathrm{g} / \mathrm{ml}(121.43 \pm 5.67 \%), 300 \mu \mathrm{g} /$ $\mathrm{ml}(118.44 \pm 3.48 \%)$ and $400 \mu \mathrm{g} / \mathrm{ml}(119.81 \pm 2.77 \%)$ for $48 \mathrm{~h}$ was enhanced in a dose-dependent manner compared to that of the cells treated with $\mathrm{CoCl}_{2}$ only $(100 \pm 0.00 \%)(\mathrm{P}<0.01)$. Cell 

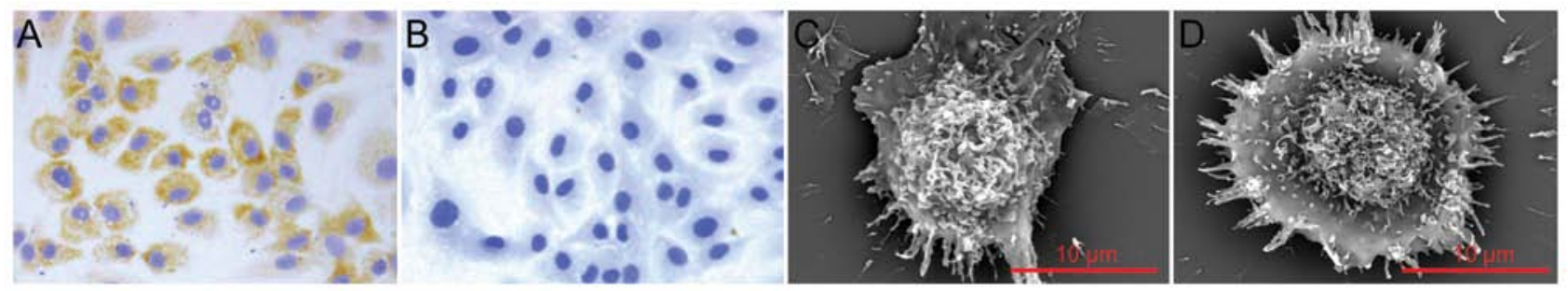

Figure 2. Identification of chondrocytes. (A) Chondrocytes were examined by type II collagen immunohistochemical staining. Cells positive for type II collagen (chondrocytes) were stained brown in the cytoplasm. (B) Cells negative for type II collagen (not chondrocytes) were not stained. (C and D) Morphology of chondrocytes was observed under a scanning electron microscope.
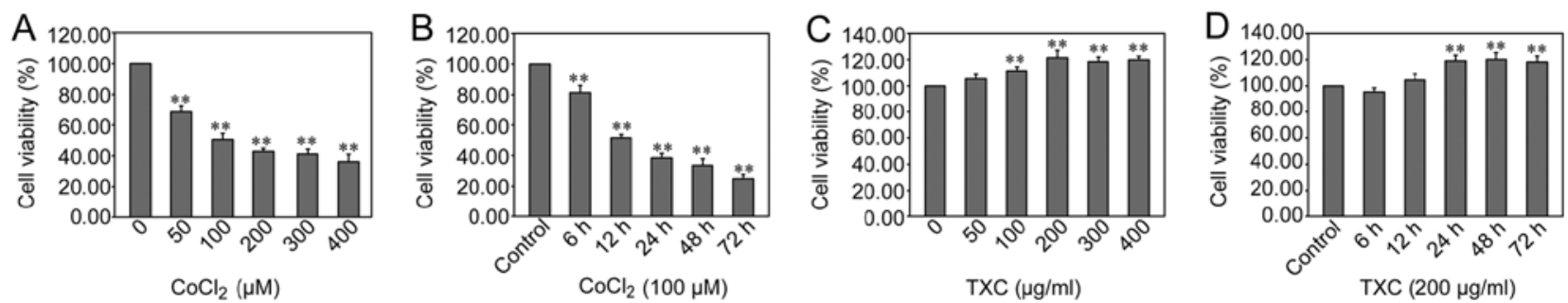

Figure 3. Tougu Xiaotong capsule (TXC) enhances the viability of cobalt chloride $\left(\mathrm{CoCl}_{2}\right)$-exposed chondrocytes. (A) Chondrocytes were treated with various concentrations of $\mathrm{CoCl}_{2}$ for $24 \mathrm{~h}$. (B) Chondrocytes were treated with $100 \mu \mathrm{M} \mathrm{CoCl}_{2}$ for the indicated periods of time. (C) $\mathrm{CoCl}_{2}$-exposed chondrocytes were treated with various concentrations of TXC for $48 \mathrm{~h}$. (D) $\mathrm{CoCl}_{2}$-exposed chondrocytes were treated with $200 \mu \mathrm{g} / \mathrm{ml} \mathrm{TXC} \mathrm{for} \mathrm{the} \mathrm{indicated} \mathrm{periods} \mathrm{pf} \mathrm{time.} \mathrm{Data}$ are the means \pm standard deviation (SD) and $\mathrm{SD}$ is shown as vertical bars, ${ }^{* * *} \mathrm{P}<0.01$, compared to untreated cells.
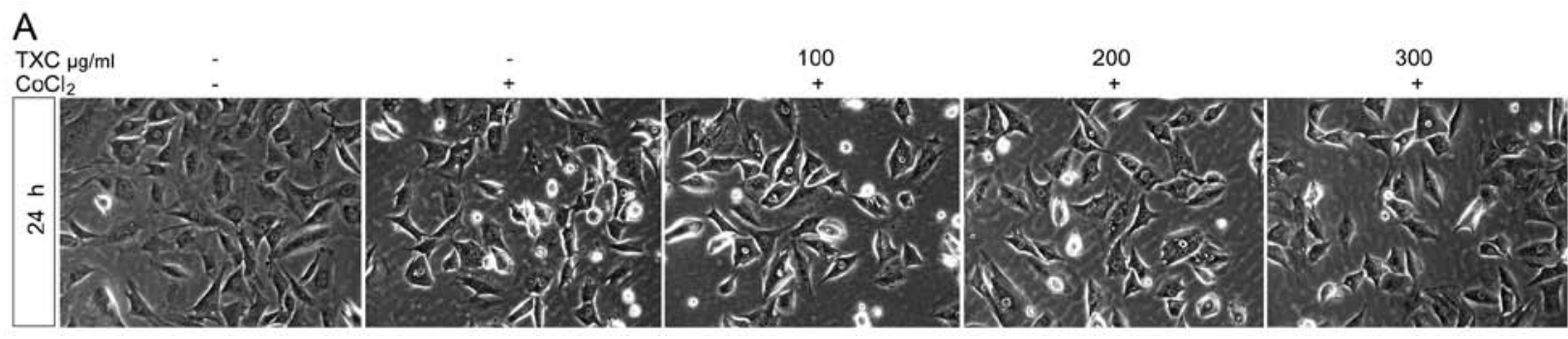

B
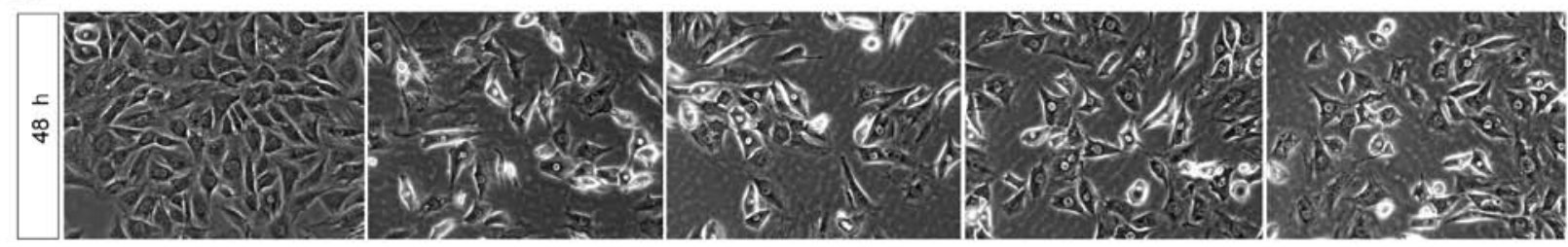

Figure 4. Tougu Xiaotong capsule (TXC) affects the morphology of cobalt chloride $\left(\mathrm{CoCl}_{2}\right)$-exposed chondrocytes (x200). The morphological changes of $\mathrm{CoCl}_{2}$-exposed chondrocytes treated with various concentrations of TXC for 24 and $48 \mathrm{~h}$ were observed under a phase-contrast microscope.

viability gradually increased with the increase in the duration of treatment in the $\mathrm{CoCl}_{2}$-exposed chondrocytes treated with $200 \mu \mathrm{g} / \mathrm{ml}$ TXC for different periods of time (Fig. 3D), indicating that TXC promoted the survival of $\mathrm{CoCl}_{2}$-exposed chondrocytes.

TXC enhances chondrocyte survival by promoting cell autophagy. TXC has been reported to delay cartilage degradation by activating chondrocyte autophagy in vivo; however, it remains to be seen whether TXC enhances chondrocyte survival by increasing the expression of Atg genes. To verify the effects of TXC on chondrocyte autophagy, we observed the morphology of $\mathrm{CoCl}_{2}$-exposed chondrocytes treated with or without TXC under a phase-contrast microscope. As shown in Fig. 4, many of the chondrocytes treated with $\mathrm{CoCl}_{2}$ for 24 and $48 \mathrm{~h}$ became rounded and shrunken, and were detached from each other or floated in the medium, compared to the untreated chondrocytes that showed densely disorganized multilayers. However, TXC inhibited the changes in the morphology of $\mathrm{CoCl}_{2}$-exposed chondrocytes, suggesting that TXC enhanced chondrocyte survival by inhibiting the $\mathrm{CoCl}_{2}$-induced autophagic death.

To explore the role of TXC in chondrocyte autophagy, the mRNA and protein expression of Atg genes in $\mathrm{CoCl}_{2}$-exposed chondrocytes treated with or without TXC was examined by 

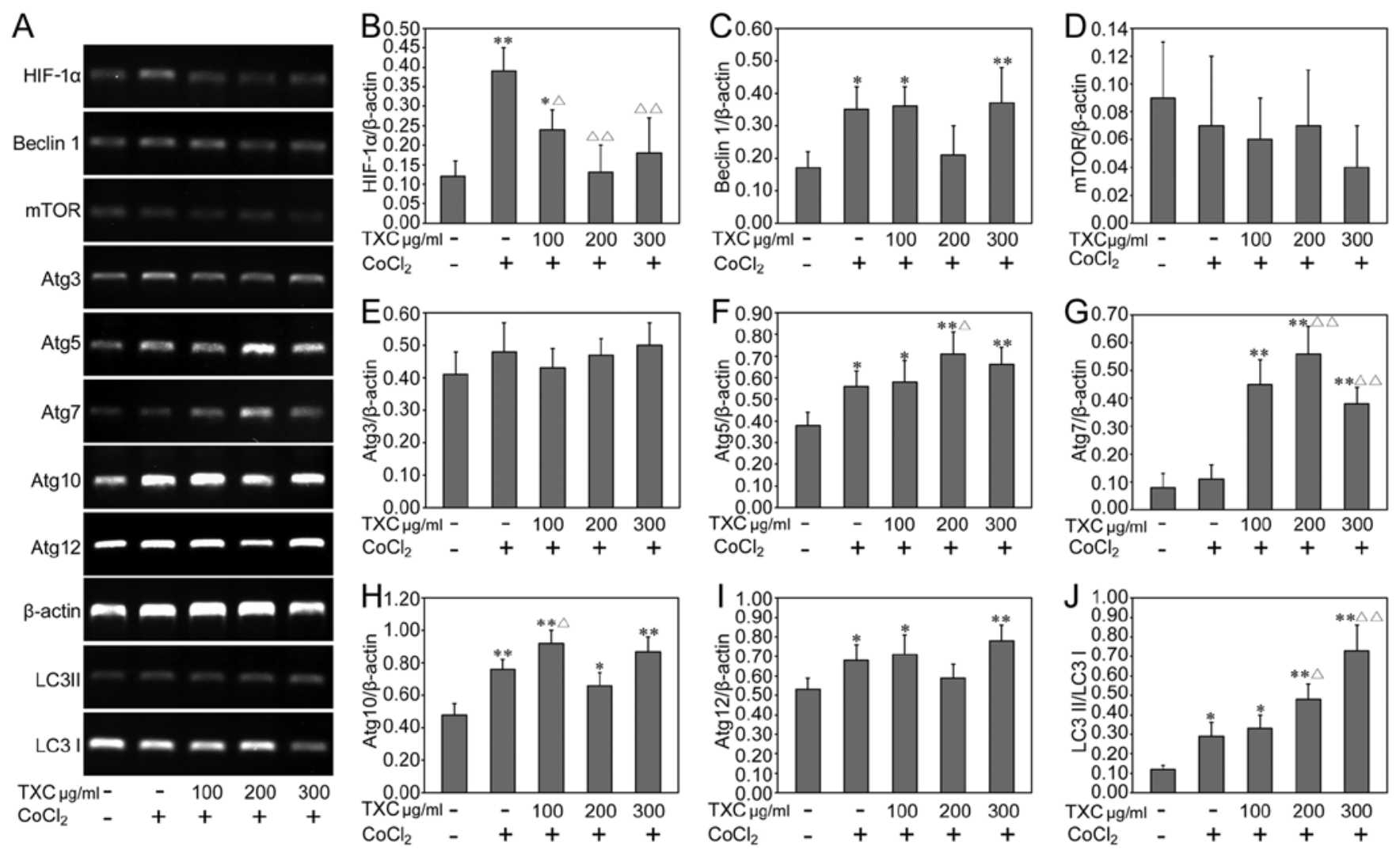

Figure 5. Tougu Xiaotong capsule (TXC) regulates the mRNA expression of autophagy-related (Atg) genes in cobalt chloride (CoCl $)_{2}$-exposed chondrocytes. (A) mRNA expression of Atg genes in $\mathrm{CoCl}_{2}$-exposed chondrocytes treated with or without TXC measured by RT-PCR. The mRNA expression of (B) hypoxia-inducible factor-1 $\alpha(\mathrm{HIF}-1 \alpha)$, (C) beclin 1, (D) mammalian target of rapamycin (mTOR), (E) Atg3, (F) Atg5, (G) Atg7, (H) Atg10, (I) Atg12 and $(\mathrm{J})$ microtubule-associated protein 1 light chain 3 (LC3) II/LC3 I. $\beta$-actin was used as the internal control for the quantification analysis. Data are the means \pm standard deviation (SD) and SD is shown as vertical bars, ${ }^{*} \mathrm{P}<0.05,{ }^{* *} \mathrm{P}<0.01$, compared to untreated cells; ${ }^{\Delta} \mathrm{P}<0.05,{ }^{\Delta \Delta} \mathrm{P}<0.01$, compared to ${ }^{\circ}{ }^{2} l_{2}-$ exposed-chondrocytes.

RT-PCR and western blot analysis, respectively. The results revealed that the mRNA expression of beclin 1, Atg3, Atg5, Atg7, Atg10, Atg12 and LC3 II/LC3 I in the chondrocytes treated with TXC increased, compared to the untreated cells $(\mathrm{P}<0.05, \mathrm{P}<0.01)$ (Fig. 5). The protein expression levels of these Atg genes were similar to their respective mRNA expression levels (Fig. 6), suggesting that TXC promoted chondrocyte autophagy by regulating the Atg12/LC3 conjugation systems.

TXC promotes the formation of autophagosomes in $\mathrm{CoCl}_{2}$ exposed-chondrocytes. We examined the ultrastructural changes of $\mathrm{CoCl}_{2}$-exposed chondrocytes treated with or without TXC by TEM. The results revealed that the nuclei of the untreated chondrocytes had a normal appearance, a Golgi apparatus, rough endoplasmic reticulum (ERr), vesicles and mitochondria (Fig. 7A). By contrast, the chondrocytes treated with TXC displayed an increase in the number of autophagosomes (Fig. 7B-H). These autophagosomes were recognized as double-membrane structures with contents ranging from degenerated organelles to granular cytoplasm, protein aggregates and endoplasmic reticulum. The fusion of autophagosomes with lysosomes was also observed (Fig. 7G and $\mathrm{H}$ ), indicating that TXC enhanced chondrocyte autophagy by promoting the formation of autophagosomes in $\mathrm{CoCl}_{2}$ exposed chondrocytes.

\section{Discussion}

The present study systematically investigated the effects of TXC on $\mathrm{CoCl}_{2}$-induced chondrocyte autophagic death in vitro. Our results clearly demonstrated that TXC enhanced the viability of chondrocytes exposed to $\mathrm{CoCl}_{2}$ and increased the formation of autophagosomes in the $\mathrm{CoCl}_{2}$-exposed chondrocytes by upregulating the expression of beclin 1, Atg3, Atg5, Atg7, Atg10, Atg12 and LC3 II/LC3 I. Taken together, these results indicate that $\mathrm{TXC}$ is a potential therapeutic agent for the reduction of cartilage degradation that occurs in OA.

Current treatments for the management of OA do not reverse the degradation process of articular cartilage. Non-steroidal anti-inflammatory drugs (NSAIDs) have been used in the treatment of OA for the past several years; however, their therapeutic effects remain unsatisfactory due to the serious adverse side-effects, such as gastrointestinal and cardiovascular diseases (21-23). Accordingly, the development of novel drugs from natural herbs, which can provide cartilage protection and be safely used in the prolonged treatment of OA, is required. TXC has been used to control tidemark replication and cartilage degradation by inhibiting chondrocyte apoptosis and promoting chondrocyte autophagy $(18,19)$. Therefore, in the present study, we sought to determine the efficacy of TXC on chondrocyte autophagy, as a chondroprotective agent. 

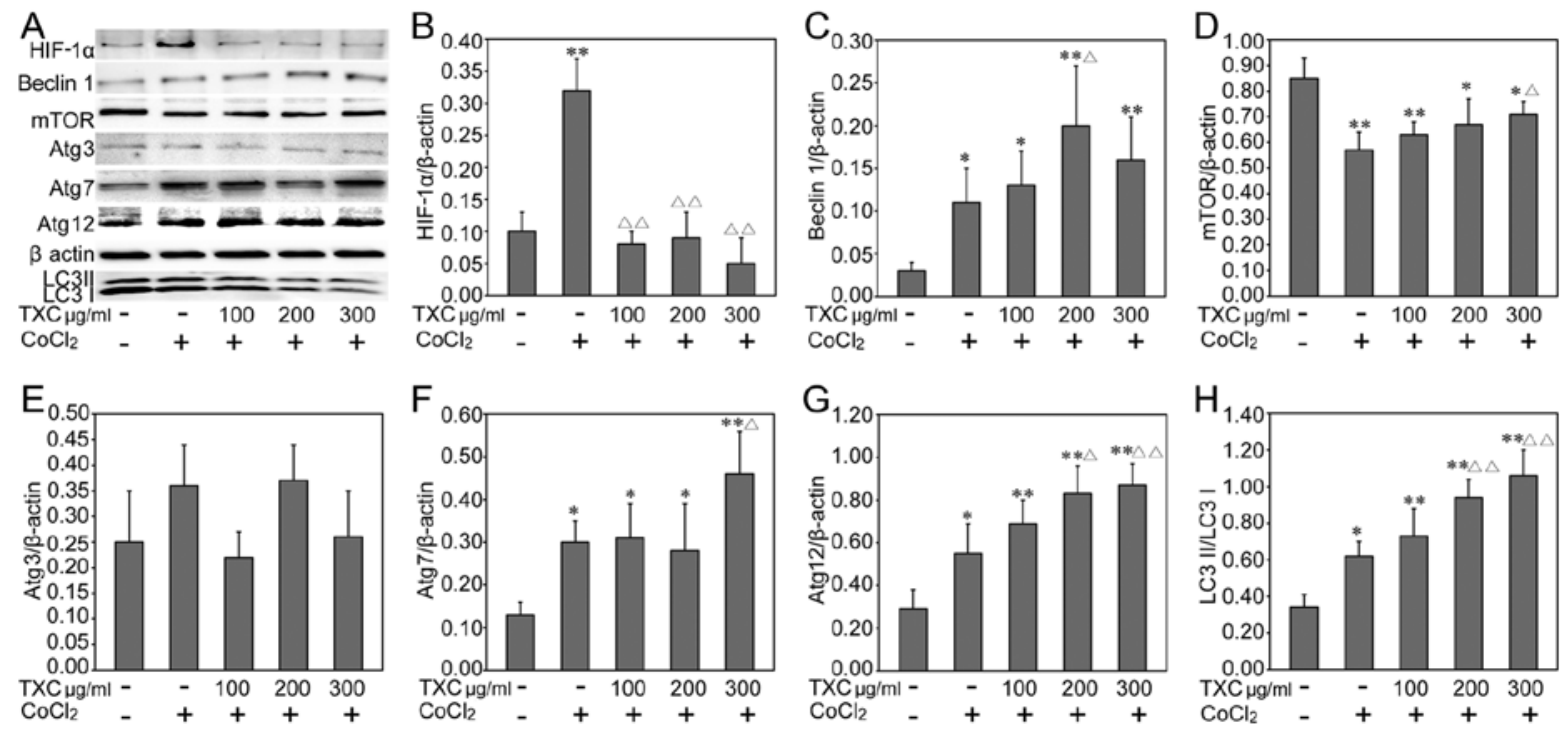

Figure 6. Tougu Xiaotong capsule (TXC) regulates the protein expression of autophagy-related (Atg) genes in cobalt chloride $\left(\mathrm{CoCl}_{2}\right)$-exposed chondrocytes (A) Protein expression of Atg genes in $\mathrm{CoCl}_{2}$-exposed chondrocytes measured by western blot analysis. Protein levels of (B) hypoxia-inducible factor-1 $\alpha$ (HIF1 $\alpha$ ), (C) beclin 1, (D) mammalian target of rapamycin (mTOR), (E) Atg3, (F) Atg7, (G) Atg12, and (H) microtubule-associated protein 1 light chain 3 (LC3) II/ LC3 I. $\beta$-actin was used as the internal control for the quantification analysis. Data are the means \pm standard deviation (SD) and SD is shown as vertical bars, ${ }^{*} \mathrm{P}<0.05,{ }^{* *} \mathrm{P}<0.01$, compared to untreated cells; ${ }^{\wedge} \mathrm{P}<0.05,{ }^{\Delta} \mathrm{P}<0.01$, compared to $\mathrm{CoCl}_{2}$-exposed-chondrocytes.
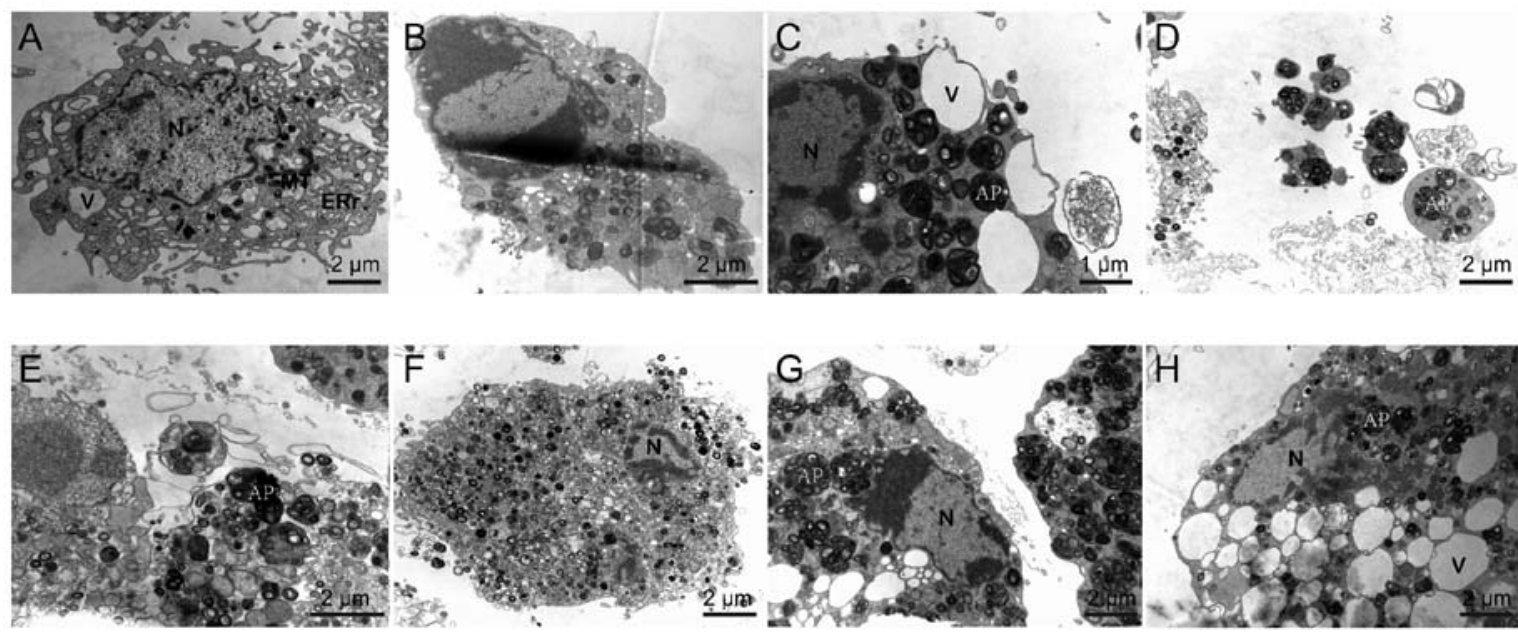

Figure 7. Tougu Xiaotong capsule (TXC) promotes the formation of autophagosomes in cobalt chloride $\left(\mathrm{CoCl}_{2}\right)$-exposed chondrocytes. (A) Chondrocytes with a small portion of rough endoplasmic reticulum (ERr), mitochondria (MT) and some vesicles (V) are visible in the cytoplasm; the nucleus (N) contains heterochromatin and euchromatin. (B) Apoptotic bodies were observed in the $\mathrm{CoCl}_{2}$-exposed chondrocytes. $(\mathrm{C}-\mathrm{H}) \mathrm{CoCl}_{2}$-exposed chondrocytes treated with or without TXC displayed a high number of autophagosomes (AP).

Excessive mechanical loading of articular cartilage causes damage to chondrocytes and the ECM, and initiates a pathogenetic process that occurs due to abnormal chondrocyte activation and results in the spreading of chondrocyte death and damage to the ECM beyond the initial area that was exposed to the highest mechanical load $(24,25)$. The understanding of the mechanisms involved in these cellular changes may provide the potential to identify targets for pharmacological interventions in order to attenuate or prevent the subsequent development of OA. In the articular cartilage, autophagy activated by different types of stress has been shown to be a constitutively active and protective process for the survival of chondrocytes (26). A previous study reported that the expression of 3 markers for different stages of autophagy, including uncoordinated-51-like kinase (ULK), beclin 1 and LC3, was decreased in the cartilage of patients with OA and in a mouse model of OA (8).

In the present study, we used a $\mathrm{CoCl}_{2}$-induced model of cell autophagic death to assess the molecular mechanisms responsible for the promoting effects of TXC on chondrocyte autophagy in vitro. In order to determine the inducer concentration of $\mathrm{CoCl}_{2}$ in chondrocytes, cell viability was examined. Following exposure to $\mathrm{CoCl}_{2}$ at various concentrations and for different periods of time, cell viability was inhibited due to the $\mathrm{CoCl}_{2}$-induced autophagic death as shown by MTT assay. Our results revealed that $\mathrm{CoCl}_{2}$ induced autophagic death in a dose- and time-dependent manner. The changes observed in cell morphology suggested that the cells underwent autophagic death $24 \mathrm{~h}$ following incubation with 
the concentration of $\mathrm{CoCl}_{2}$ selected based on the results of MTT assay. To determine the effective concentration of TXC on the viability of $\mathrm{CoCl}_{2}$-exposed chondrocytes, MTT assay was performed. These results indicated that TXC enhanced chondrocyte survival, as shown by the increased cell viability.

Autophagy, which is in part related to the reduced expression of autophagic regulators, is compromised in osteoarthritic cartilage. In articular cartilage, autophagy does not only occur in response to mechanical injury, but is also deficient with aging $(8,27)$. Autophagy is characterized by the formation of autophagosomes and their fusion with lysosomes. At the late stage of autophagy, lysosomes fuse with and release lysosomal enzymes into the autophagosome to degrade its contents (28). Atg genes control the autophagy process leading to the induction and nucleation of autophagic vesicles, their fusion and expansion with lysosomes, following enzymatic degradation and recycling $(29,30)$. Atg12 undergoes an ubiquitin-like conjugation to Atg5 through an internal lysine residue and a $\mathrm{COOH}$-terminal glycine, respectively. This process is activated by the Atg7 protein, which is homologous to the E1 family of ubiquitin-activating enzymes, and Atg10, which functions as a protein-conjugating enzyme (31). The Atg12-Atg5 conjugates recruit Atg16 dimers. Atg16 is a bivalent molecule, which leads to the formation of large multimeric complexes, and these are thought to play a key role in the nucleation of both cytoplasmto-vacuole targeting vesicles and autophagosomes (32). The number of lysosomes increases in the process of autophagy, accompanied by an increased expression of beclin 1 and LC3 (33). Beclin 1, forming a complex with type III PI3 kinase and Vps34, participates in the nucleation of the autophagic vesicle (19). The involvement of LC3 in the protein conjugation system is required in the expansion of the autophagosome. There are 2 forms of LC3, including LC3 I, in the cytoplasm and LC3 II bound to the autophagosome membrane. During autophagy, LC3 I is converted to LC3 II by the ubiquitin-like system. Thus, the level of LC3 II and the ratio of LC3 II to LC3 I closely reflect the extent of autophagy (34).

To gain insight into the mechanisms responsible for the effects of TXC on $\mathrm{CoCl}_{2}$-induced autophagic death, the expression of mTOR and HIF-1 $\alpha$, as modulators of autophagy, was assessed in chondrocytes. mTOR plays an important role in multiple cellular functions, such as cell metabolism, proliferation and autophagy (35). All Atg genes have been shown to act downstream of mTOR. HIF-1 $\alpha$, a heterodimeric transcription factor that mediates adaptive responses to hypoxia, serves to regulate both autophagy and apoptosis (36). The present results indicated that the expression of beclin 1, Atg3, Atg5, Atg7, Atg10, Atg12 and LC3 II/LC3 I in the $\mathrm{CoCl}_{2}$-exposed chondrocytes treated with or without TXC significantly increased, compared to the untreated cells. In addition, ultrastructural analysis revealed that the chondrocytes treated with TXC contained more autophagosomes than the untreated cells, suggesting that TXC increases the formation of autophagosomes in chondrocytes to clear the $\mathrm{CoCl}_{2}$-induced autophagic death.

Based on these results, we hypothesized that TXC promotes chondrocyte autophagy by regulating the Atg12/ LC3 conjugation systems. Since autophagy serves to delay the onset of apoptosis, experiments are curently in progress in order to explore whether there is a direct association between the induction of autophagy and apoptosis.

\section{Acknowledgements}

This study was supported by the National Natural Science Foundation of China (grant no. 81102609), the Key Project of Fujian Provincial Department of Science and Technology (grant no. 2012Y0046), the Natural Science Foundation of Fujian Province (grant no. 2011J05074) and the Young Talent Scientific Research Project of Fujian Province Universities (grant no. JA12165).

\section{References}

1. Taniguchi N, Caramés B, Ronfani L, et al: Aging-related loss of the chromatin protein HMGB2 in articular cartilage is linked to reduced cellularity and osteoarthritis. Proc Natl Acad Sci USA 106: 1181-1186, 2009.

2. Ruiz-Romero C, Calamia V, Mateos J, et al: Mitochondrial dysregulation of osteoarthritic human articular chondrocytes analyzed by proteomics: a decrease in mitochondrial superoxide dismutase points to a redox imbalance. Mol Cell Proteomics 8: 172-189, 2009.

3. Li XH, Liang WN and Liu XX: Clinical observation on curative effect of dissolving phlegm-stasis on 50 cases of knee osteoarthritis. J Tradit Chin Med 30: 108-112, 2010.

4. Loeser RF: Aging and osteoarthritis: the role of chondrocyte senescence and aging changes in the cartilage matrix. Osteoarthritis Cartilage 17: 971-979, 2009.

5. Pennock AT, Robertson CM, Emmerson BC, Harwood FL and Amiel D: Role of apoptotic and matrix-degrading genes in articular cartilage and meniscus of mature and aged rabbits during development of osteoarthritis. Arthritis Rheum 56: 1529-1536, 2007.

6. Lotz MK and Caramés B: Autophagy and cartilage homeostasis mechanisms in joint health, aging and OA. Nat Rev Rheumatol 7: 579-587, 2011.

7. Caramés B, Hasegawa A, Taniguchi N, Miyaki S, Blanco FJ and Lotz M: Autophagy activation by rapamycin reduces severity of experimental osteoarthritis. Ann Rheum Dis 71: 575-581, 2012.

8. Caramés B, Taniguchi N, Otsuki S, Blanco FJ and Lotz M: Autophagy is a protective mechanism in normal cartilage, and its aging-related loss is linked with cell death and osteoarthritis. Arthritis Rheum 62: 791-801, 2010.

9. Levine B and Kroemer G: Autophagy in the pathogenesis of disease. Cell 132: 27-42, 2008.

10. Cecconi $\mathrm{F}$ and Levine B: The role of autophagy in mammalian development: cell makeover rather than cell death. Dev Cell 15: 344-357, 2008.

11. Mizushima N, Levine B, Cuervo AM and Klionsky DJ: Autophagy fights disease through cellular self-digestion. Nature 451:1069-1075, 2008.

12. Mathew R, Karp CM, Beaudoin B, et al: Autophagy suppresses tumorigenesis through elimination of p62. Cell 137: 1062-1075, 2009.

13. Komatsu M, Waguri S, Ueno T, et al: Impairment of starvation-induced and constitutive autophagy in Atg7-deficient mice. J Cell Biol 169: 425-434, 2005.

14. Hara T, Nakamura K, Matsui M, et al: Suppression of basal autophagy in neural cells causes neurodegenerative disease in mice. Nature 441: 885-889, 2006.

15. Yang Q and Guan KL: Expanding mTOR signaling. Cell Res 17: 666-681, 2007.

16. Wullschleger $\mathrm{S}$, Loewith $\mathrm{R}$ and Hall $\mathrm{MN}$ : TOR signaling in growth and metabolism. Cell 124: 471-484, 2006.

17. Cuervo AM: Autophagy and aging: keeping that old broom working. Trends Genet 24: 604-612, 2008.

18. Li XH, Wu MX, Ye HZ, et al: Experimental study on the suppression of sodium nitroprussiate-induced chondrocyte apoptosis by Tougu Xiaotong capsule-containing serum. Chin J Integr Med 17: 436-443, 2011.

19. Li X, Lang W, Ye H, et al: Tougu Xiaotong capsule inhibits the tidemark replication and cartilage degradation of papain-induced osteoarthritis by the regulation of chondrocyte autophagy. Int J Mol Med 31: 1349-1356, 2013.

20. Li X, Ye H, Yu F, et al: Millimeter wave treatment promotes chondrocyte proliferation via G1/S cell cycle transition. Int J Mol Med 29: 823-831, 2012. 
21. Schnitzer TJ, Burmester GR, Mysler E, et al: Comparison of lumiracoxib with naproxen and ibuprofen in the Therapeutic Arthritis Research and Gastrointestinal Event Trial (TARGET), reduction in ulcer complications: randomised controlled trial. Lancet 364: 665-674, 2004.

22. Lichtenberger LM, Zhou Y, Dial EJ and Raphael RM: NSAID injury to the gastrointestinal tract: evidence that NSAIDs interact with phospholipids to weaken the hydrophobic surface barrier and induce the formation of unstable pores in membranes. J Pharm Pharmacol 58: 1421-1428, 2006.

23. Farkouh ME, Greenberg JD, Jeger RV, et al: Cardiovascular outcomes in high risk patients with osteoarthritis treated with ibuprofen, naproxen or lumiracoxib. Ann Rheum Dis 66: 764-770, 2007.

24. Otsuki S, Brinson DC, Creighton L, et al: The effect of glycosaminoglycan loss on chondrocyte viability: a study on porcine cartilage explants. Arthritis Rheum 58: 1076-1085, 2008.

25. D'Lima DD, Hashimoto S, Chen PC, Colwell CW Jr and Lotz MK Human chondrocyte apoptosis in response to mechanical injury. Osteoarthritis Cartilage 9: 712-719, 2001.

26. Goldring SR and Goldring MB: Clinical aspects, pathology and pathophysiology of osteoarthritis. J Musculoskelet Neuronal Interact 6: 376-378, 2006

27. Caramés B, Taniguchi N, Seino D, Blanco FJ, D'Lima D and Lotz M: Mechanical injury suppresses autophagy regulators and pharmacologic activation of autophagy results in chondroprotection. Arthritis Rheum 64: 1182-1192, 2012.
28. Kimura S, Noda T and Yoshimori T: Dynein-dependent movement of autophagosomes mediates efficient encounters with lysosomes. Cell Struct Funct 33: 109-122, 2008.

29. Srinivas V, Bohensky J, Zahm AM and Shapiro IM: Autophagy in mineralizing tissues: microenvironmental perspectives. Cell Cycle 8: 391-393, 2009.

30. Cajee UF, Hull R and Ntwasa M: Modification by ubiquitin-like proteins: significance in apoptosis and autophagy pathways. Int J Mol Sci 13: 11804-11831, 2012.

31. Klionsky DJ and Emr SD: Autophagy as a regulated pathway of cellular degradation. Science 290: 1717-1721, 2000.

32. Abeliovich $\mathrm{H}$ and Klionsky DJ: Autophagy in yeast: mechanistic insights and physiological function. Microbiol Mol Biol Rev 65: 463-479, 2001.

33. Ohsumi Y and Mizushima N: Two ubiquitin-like conjugation systems essential for autophagy. Semin Cell Dev Biol 15: 231-236, 2004.

34. Caramés B, Kiosses WB, Akasaki Y, et al: Glucosamine activates autophagy in vitro and in vivo. Arthritis Rheum 65: 1843-1852, 2013.

35. Srinivas V, Bohensky J and Shapiro IM: Autophagy: a new phase in the maturation of growth plate chondrocytes is regulated by HIF, mTOR and AMP kinase. Cells Tissues Organs 189: 88-92, 2009.

36. Bohensky J, Shapiro IM, Leshinsky S, Terkhorn SP, Adams CS and Srinivas V: HIF-1 regulation of chondrocyte apoptosis: induction of the autophagic pathway. Autophagy 3: 207-214, 2007. 